\title{
What Are the Critical Histologic Features in the Diagnosis of Ulcerative Colitis?
}

\author{
Henry D. Appleman, MD
}

$\mathrm{U}$

lcerative colitis (UC) is a chronic disease, so changes of chronicity are essential for histologic diagnosis. These chronic changes can be separated into 2 components. The first and most important component is chronic inflammation, which is mainly an increase in plasma cells (plasmacytosis) in the lamina propria. The colonic mucosa is normally in a state of chronic inflammation, so plasma cells are a normal constituent of the lamina propria, with more such cells on the right side and fewer on the left. Therefore, the determination of whether plasmacytosis is present depends on the pathologist's knowing these normal variations and knowing the specific sites of the biopsies. Eosinophils are often also increased numerically in chronic colonic inflammation, but there is also a wide range of normal numbers of eosinophils in the lamina propria that may be geographic dependent, at least in part. In 1 study of eosinophil numbers in the lamina propria of normal left colon biopsies, there was a gradual increase from north to south along both coasts. ${ }^{1}$ The normal eosinophil counts were about 30 times higher in New Orleans than in Boston. It is likely that there is also variability in normal individual eosinophil counts as well. Thus, determination of eosinophilia in colonic lamina propria has to take these geographic and individual variations into account.

The second component of chronicity is distortion. The normal colonic mucosa has uniform parallel crypts that are evenly spaced as a result of uniformity in the amount of lamina propria that separates them. The surface is flat. Destruction of the normal structures, including crypts and surface mucosa, is followed by regeneration that assumes whatever contours are available. This results in a variety of altered shapes and sizes of crypts and changes in surface contours that vary from undulating to villiform.

This combination of chronic inflammation and distortion, diffusely involving some length of colon, is required in at least 1 set of biopsies sometime during the course of the disease to prove histologically that the patient has UC. In children the chronic inflammation is present, but often distortion is less evident than in adults. ${ }^{2,3}$ Furthermore, since

From the Department of Pathology, University of Michigan, University Hospitals, Michigan, USA.

Copyright (C) 2008 Crohn's \& Colitis Foundation of America, Inc. DOI 10.1002/ibd.20586

Published online in Wiley InterScience (www.interscience.wiley.com). other diseases, such as Crohn's colitis and the microscopic colitides, including the collagenous and lymphocytic variants, have variable amounts of these two features, more proof is needed that the patient has UC. This proof comes in the form of characteristic clinical features and endoscopic findings of diffuse disease covering stretches of mucosa from rectum only to total colon, and biopsies that sample the entire areas of endoscopic disease as well as biopsies taken from proximal to the endoscopic disease to determine the extent. Full-blown Crohn's colitis has both distortion and plasmacytosis, but the disease is patchy and intense, both histologically and endoscopically, and there is almost always evidence of small bowel involvement. Crohn's disease (CD) also often has tiny microscopic foci of inflammation throughout the gastrointestinal (GI) tract, but these are not evidence of important structural disease. Lymphocytic and collagenous colitis have diffuse plasmacytosis, but little distortion, and the mucosa looks normal endoscopically.

UC is not a static disease but is a disease that constantly changes as a result of exacerbations and remissions, some of which are treatment-induced at the clinical, endoscopic, and histologic levels. As a result, how UC appears at any given moment depends on whether the disease is in an exacerbation or a remission, and where in that exacerbation or remission it is seen. We know that with time and treatment the UC changes can resolve. The inflammation subsides and the mucosa remodels, often returning to normal. Also, at various times during its evolutions in some patients the histologic and endoscopic disease may become focal or patchy. In addition, significant rectal inflammation can heal and become endoscopically and histologically normal. ${ }^{4-6}$. In these settings the biopsy changes are no longer diagnostic and may even resemble Crohn's colitis because of the patchy distribution and normal rectum. Similar changes are found in colectomy specimens. However, in many colectomy specimens the entire mucosa looks normal or near normal, probably because of intense medical treatment prior to resection.

Finally, the chronic changes may be accompanied by active inflammation, that is, neutrophils invading crypt epithelium (cryptitis), sometimes resulting in ulcers involving parts of the crypt epithelium as well as pus filling and distending crypts (crypt abscesses). However, the changes of activity are not required for the diagnosis of UC; the chronic changes are required. 
In summary, there are mucosal changes, both in biopsies and in colectomy specimens, that allow for the diagnosis of UC. The required changes are chronic inflammation. Architectural distortion is not required, but it is helpful for diagnosis. However, biopsies that have these changes are only diagnostic when found in the characteristic endoscopic and clinical settings.

\section{REFERENCES}

1. Pascal RR, Gramlich TL, Parker KM, et al. Geographic variations in eosinophil concentration in normal colonic mucosa. Mod Pathol. 1997; 10:363-365.
2. Robert ME, Tang L, Hao LM, et al. Patterns of inflammation in mucosal biopsies of ulcerative colitis. Perceived differences in pediatric populations are limited to children younger than 10 years. Am J Surg Pathol. 2004;28:183-189.

3. Washington K, Greenson JK, Montgomery E, et al. Histopathology of ulcerative colitis in initial rectal biopsy in children. Am J Surg Pathol. 2002;26:1441-1449.

4. Bernstein CN, Shanahan F, Anton PA, et al. Patchiness of mucosal inflammation in treated ulcerative colitis: a prospective study. Gastrointest Endosc. 1995;42:232-237.

5. Kim B, Barnett JL, Kleer CS, et al. Endoscopic and histologic patchiness in treated ulcerative colitis. Am J Gastroenterol. 1999;94:3258-3262.

6. Kleer CG, Appelman HD. Ulcerative colitis: patterns of involvement in colorectal biopsies and changes with time. Am J Surg Pathol. 1998;22: 983-989. 